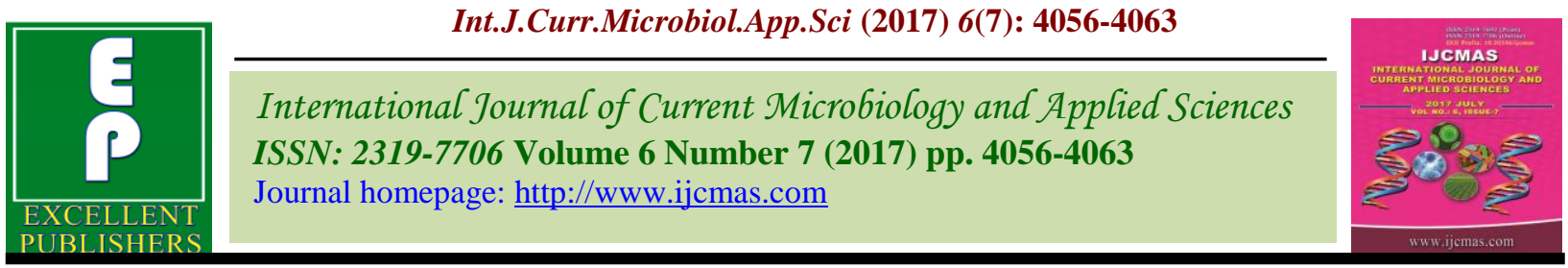

Original Research Article

https://doi.org/10.20546/ijcmas.2017.607.420

\title{
Elucidation of rRNA Secondary Structure and Phylogenetic Analysis of Salmonella enterica based on 16S RNA
}

\author{
Priyanka Chandra ${ }^{1,2}$ and Amaresh Chandra ${ }^{2 *}$ \\ ${ }^{1}$ ICAR-Central Soil Salinity Research Institute, Karnal, India \\ ${ }^{2}$ ICAR- Indian Institute of Sugarcane Research, Lucknow, India \\ *Corresponding author
}

\begin{tabular}{|c|c|}
\hline & A B S T R A C T \\
\hline $\begin{array}{l}\text { Ke y w or d s } \\
\text { Identification, } \\
\text { Salmonella } \\
\text { enterica, } \\
\text { 16S RNA, } \\
\text { Biochemical tests, } \\
\text { phylogenetic tree. }\end{array}$ & \multirow{3}{*}{$\begin{array}{l}\text { Salmonella enterica is a Gram-negative, facultatively anaerobic rod and a } \\
\text { member of the Enterobacteriaceae. There are currently more than } 2500 \\
\text { serovars of } S \text {. enterica. S. enterica is an important cause of foodborne } \\
\text { illness in the developing countries and causes sporadic outbreaks of } \\
\text { Salmonellosis in the developed countries. In the present study the isolate } \\
\text { was confirmed on the basis of Gram staining and was subjected to various } \\
\text { biochemical tests. Identification of bacterial strain was carried out using } \\
\text { 16SrRNA gene sequencing. The isolate was found to be Salmonella } \\
\text { enterica and most similar to Salmonella enterica subsp. enterica serovar } \\
\text { Panama str. ATCC 7378. RNA secondary structure prediction has been } \\
\text { performed. The } 16 \mathrm{~S} \text { RNA gene sequence obtained was used to deduce the } \\
\text { secondarv structure of RNA ViennaRNA Web Services. }\end{array}$} \\
\hline Article Info & \\
\hline $\begin{array}{l}\text { Accepted: } \\
\text { 29 June } 2017 \\
\text { Available Online: } \\
\text { 10 July } 2017\end{array}$ & \\
\hline
\end{tabular}

\section{Introduction}

Salmonella spp. are ubiquitous enteric bacteria. These are gram-negative rods and etiologic agents of food-borne salmonellosis, typhoid and paratyphoid fevers. The genus Salmonella comprises a group of Gramnegative bacilli belonging to the family Enterobacteriaceae and contains a number of closely related organisms. The genus consists of two species, $S$. enterica and $S$. bongeri. $S$. enterica has been further subdivided into over 2500 serotypes that can be differentiated by the Kauffman-White scheme, which is based on the serologic identification of $\mathrm{O}$ (somatic) and $\mathrm{H}$ (flagellar) antigens. Approximately 1400 of the serotypes have been reported to cause gastroenteritis in humans, while only a handful are capable of causing typhoid, a potentially fatal systemic infection (Leekitcharoenphon et al., 2016).

S. enterica includes six subspecies on the basis of chromosomal DNA hybridization and multilocus enzyme electrophoresis are as follows: S. enterica subsp. enterica subspecies (I), S. enterica subsp. salamae (II), S. enterica subsp. arizona (IIIa), S. enterica subsp. diarizona (IIIb), S. enterica subsp. indica (VI), S. enterica subsp. houtenae (IV). The majority of these serotypes belong to $S$. 
enterica subspecies I; these serotypes also cause most infections in humans and warmblooded animals (Porwollik et al., 2003). Whole genome comparative analyses of Salmonella serotypes have shown that approximately $90 \%$ of the genome is conserved, and there is on average about $97 \%$ sequence identity when comparing homologous genes. However, for laboratories performing routine public health surveillance for detection and identification of Salmonella, The need for the development of rapid and accurate detection methods for Salmonella has been intensified in recent years due to increasing incidence of salmonellosis in industrialised countries over the past decades. Since the traditional microbiological method for the detection of Salmonella requires up to 5-7 days, and involves several subcultivation steps followed by biochemical and serological tests, quicker approaches have been searched for, mainly at the DNA level. A fairly large number of PCR and hybridization methods for Salmonella detection have been developed (Swaminathan et al., 2001). The present study also includes the 16s RNA sequencing for the detection.

\section{Materials and Methods}

The isolate was grown on nutrient agar plates at $37^{\circ} \mathrm{C}$ for $24-48 \mathrm{~h}$ and was maintained on nutrient agar slants and stored at $4^{\circ} \mathrm{C}$ as well as at $-80^{\circ} \mathrm{C}$ by making their suspensions in $10 \%$ (v/v) glycerol. Standard biochemical tests of the culture were performed (Godkar, 1999; Cappuccino and Sherman, 2004). For catalase test, a small amount of the culture was picked up with a sterile glass rod and immersed in $3 \mathrm{ml}$ of $3 \%(\mathrm{v} / \mathrm{v})$ hydrogen peroxide $\left(\mathrm{H}_{2} \mathrm{O}_{2}\right)$ solution. Appearance of bubbles was indicative of the catalase production. For coagulase test, a uniform suspension of the bacterial isolate was made in a drop of normal saline on a slide and was mixed with a drop of plasma. A positive reaction was indicated by visible clumping of the suspension with plasma within a minute. For nitrate reduction test, sterilized nitrate broth is inoculated with the isolate and incubated for $4 \mathrm{~h}$ at $37^{\circ} \mathrm{C}$. Nitrate reductase producing organisms reduce nitrate to nitrite, which is tested by adding one drop each of sulphanilic acid and $\alpha$-naphthylamine reagents. The broth was mixed well and the formation of pink-red colour indicated the positive reaction. For oxidase test, Two ml (2 $\mathrm{ml}$ ) of freshly prepared oxidase reagent was added to a piece of filter paper. Then a colony of the culture to be tested was smeared onto it using a sterilized glass rod. A positive reaction was indicated by an intense deep purple colour appearing within 5-10 sec. For urease test, motility indole urea (MIU) agar slants were inoculated with bacteria to be tested and incubated overnight at $37^{\circ} \mathrm{C}$. A red-pink colour of the slant was indicative of the positive test. For indole test, peptone water $(5 \mathrm{ml})$ was inoculated with the organisms to be tested and incubated at $37^{\circ} \mathrm{C}$ for $24 \mathrm{~h}$. After incubation, $0.5 \mathrm{ml}$ of Kovac's reagent was added and mixed thoroughly. After mixing, the alcoholic layer separated from aqueous layer and reddening of alcoholic layer within few minutes was indicative of the positive reaction. For methyl red test, the isolate was inoculated into $5 \mathrm{ml}$ of sterile glucose phosphate broth and incubated overnight at $37^{\circ} \mathrm{C}$. Then five drops of methyl red were added and mixed thoroughly. Bright red colour was indicative of positive test while yellow colour suggested a negative test.

For Voges-Proskauer test, the isolate was inoculated into $2 \mathrm{ml}$ of glucose phosphate peptone water and incubated at $37^{\circ} \mathrm{C}$ for $24 \mathrm{~h}$. Then $0.6 \mathrm{ml}$ of Barritt's $\mathrm{A}$ and $0.2 \mathrm{ml}$ of Barritt's B reagents were added to the test tube, mixed thoroughly and then kept at room temperature for $1 \mathrm{~h}$. Pink red colour formation demonstrated a positive reaction. For citrate 
utilization test, sterile Simmon's citrate medium was inoculated with the cultures to be tested and incubated at $35^{\circ} \mathrm{C}$ up to 4 days. Blue colour and streak of growth indicated positive result while persistence of original green colour and no growth indicated negative result. For gelatin liquefaction test, nutrient gelatin medium was inoculated with the test organism and incubated at $37^{\circ} \mathrm{C}$ for $72 \mathrm{~h}$. Then the tubes were chilled at $4^{\circ} \mathrm{C}$ for $30 \mathrm{~min}$ and results were recorded. Medium in fluid state showed a positive test while medium in solid state indicated negative test. For, hydrogen sulphide production, triple sugar iron (TSI) agar slants were streaked with inoculum, incubated at $37^{\circ} \mathrm{C}$ for 7 days and observed for the blackening caused by $\mathrm{H}_{2} \mathrm{~S}$ production (Kaur, 2010).

\section{Identification of bacterial strain using 16SrRNA gene sequencing}

The DNA template was prepared by picking an individual colony of bacterial strain, and amplification of the $16 \mathrm{~S}$ rRNA gene was carried out by the PCR . PCR amplification of DNA was performed using universal primers (9F: 5'-GAGTTTGATCCTGGC TCAG -3'; 1510R: 5'-GGCTACCTTGTTACGA-3') in a reaction mixture $(25 \mu \mathrm{l})$. The amplification program for the full-length $16 \mathrm{~S}$ rRNA gene consisted of an initial denaturation at $94{ }^{\circ} \mathrm{C}$ for $2 \mathrm{~min}$, followed by 30 cycles of denaturation at $94{ }^{\circ} \mathrm{C}$ for $2 \mathrm{~min}$, primer annealing at $55{ }^{\circ} \mathrm{C}$ for $1 \mathrm{~min}$ and primer extension at $72{ }^{\circ} \mathrm{C}$ for $2 \mathrm{~min}$, followed by a final extension at $72{ }^{\circ} \mathrm{C}$ for $10 \mathrm{~min}$, in a thermocycler. Amplified PCR products of the $16 \mathrm{~S}$ ribosomal gene were separated on $1 \%$ agarose gel in $0.5 \times$ TE (Tris-EDTA) buffer containing $2 \mu \mathrm{l}$ ethidium bromide $(20 \mathrm{mg} / \mathrm{ml})$ (Chandra and Chandra, 2016). The purified PCR product samples were sent for sequencing using universal 16S rRNA sequencing primers. The sequence results were obtained from a BLAST search, and the sequences of all the related species were retrieved to determine the exact nomenclature of the isolates. The tree is created using Weighbor with alphabet size 4 and length size 1000 .

\section{Results and Discussion}

Traditional identification of bacteria was done by biochemical and phenotypic analysis using both manual and automated systems in addition to molecular methods. The identification of bacteria on the basis of phenotypic characteristics is generally not as accurate as identification based on genotypic methods. Some biochemical techniques are rapid, however, the majority depends on microbial growth and utilization via biochemical assays that require hours to days for identification. The biochemical test of the isolate was carried out and result is described in table 1 .

Isolation and identification of Salmonella continues to be an important issue in clinical and applied microbiology. The conventional methods for the detection of Salmonella require multiple steps which may take up to 5 to 7 days (Ahmed et al., 2014). The final identification and characterization of Salmonella enterica is based on biochemical reactions followed by serotyping. However, considerable variation can be seen in the biotyping pattern. Thus, a minor proportion of presumptive $S$. enterica isolates identified by biochemical testing cannot be identified by subsequent serotyping procedures. Indeed, the API 20E diagnostic, which detects 20 biochemical reactions, is a traditional method for the identification of S. enterica and other Enterobacteriaceae. Previous studies of API $20 \mathrm{E}$ have reported both good and inaccurate sample classifications. So, rapid and more sensitive methods for the detection of Salmonella species are required. Genetic identification systems may improve Salmonella identification. PCR is an extremely sensitive test, able to amplify 
picogram quantities of DNA. In recent years, PCR methods have been successfully used to detect a number of pathogens including Salmonella (Chen et al., 2010). The rRNA genes are present in all organisms. Bacterial rRNA operons are made up of $16 \mathrm{~S}$ rRNA, 23S rRNA and 5S rRNA genes. The copy number of rRNA gene clusters varies among bacterial species. A molecular method for the detection of Salmonella enterica strains based on $16 \mathrm{~S}$ rRNA sequence analysis was developed, modified and improved by Trkov and Avgustin (2003). Likewise, Salmonella spp. 16S-23S based PCR primers were designed. The 16S rDNA and 16S-23S rDNA Internal Transcribed Spacer (ITS) region has been the most popular target for bacterial taxonomy. The ITS regions exhibit a large degree of sequence and length variation. This diversity is due in part to variations in the number and type of tRNA sequences found within the spacer. Sequence polymorphisms can be used to recognize and to discriminate between different genus and species. On the other hand, the 16S rRNA gene sequences have been widely used to assess to phylogenetic closeness among bacteria. In many case, the 16S rRNA gene sequencing has been used to study the phylogenetic relationships between species, to examine the taxonomy of different subspecies and to identify the species and the sub-species. Hence in the present study the identification of the culture was carried out through molecular technique and 16s RNA aligned sequence data of isolate is as follows:

\begin{abstract}
GGATCGTGACGGGTGAGTAATGTGCGGGGAAACTGCCCGATGGAGGGGGATAACTACTGGAAACGGTAGCT AATACCGCATAACGTCGCAAGACCAAAGAGGGGGACCTTCGGGCCTCTTGCCATCGGATGTGCCCAGATGGG ATTAGCTTGTTGGTGAGGTAACGGCTCACCAAGGCGACGATCCCTAGCTGGTCTGAGAGGATGACCAGCCAC ACTGGAACTGAGACACGGTCCAGACTCCTACGGGAGGCAGCAGTGGGGAATATTGCACAATGGGCGCAAGC CTGATGCAGCCATGCCGCGTGTATGAAGAAGGCCTTCGGGTTGTAAAGTACTTTCAGCGGGGAGGAAGGTGT TGAGGTTAATAACCTCAGCAATTGACGTTACCCGCAGAAGAAGCACCGGCTAACTCCGTGCCAGCAGCCGCG GTAATACGGAGGGTGCAAGCGTTAATCGGAATTACTGGGCGTAAAGCGCACGCAGGCGGTCTGTCAAGTCGG ATGTGAAATCCCCGGGCTCAACCTGGGAACTGCATTCGAAACTGGCAGGCTAGAGTCTTGTAGAGGGGGGTA GAATTCCAGGTGTAGCGGTGAAATGCGTAGAGATCTGGAGGAATACCGGTGGCGAAGGCGGCCCCCTGGAC AAAGACTGACGCTCAGGTGCGAAAGCGTGGGGAGCAAACAGGATTAGATACCCTGGTAGTCCACGCCGTAA ACGATGTCGACTTGGAGGTTGTGCCCTTGAGGCGTGGCTTCCGGAGCTAACGCGTTAAGTCGACCGCCTGGG GAGTACGGCCGCAAGGTTAAAACTCAAATGAATTGACGGGGGCCCGCACAAGCGGTGGAGCATGTGGTTTA ATTCGATGCAACGCGAAGAACCTTACCTGGTCTTGACATCCACGGAAGAATCCAGAGATGGATTTGTGCCTT CGGGAACCGTGAGACAGGTGCTGCATGGCTGTCGTCAGCTCGTGTTGTGAAATGTTGGGTTAAGTCCCGCAA CGAGCGCAACCCTTATCCTTTGTTGCCAGCGGTCCGGCCGGGAACTCAAAGGAGACTGCCAGTGATAAACTG GAGGAAGGTGGGGATGACGTCAAGTCATCATGGCCCTTACGACCAGGGCTACACACGTGCTACAATGGCATA TACAAAGAGAAGCGACCTCGCGAGAGCAAGCGGACCTCATAAAGTATGTCGTAGTC

CGGATTGGAGTCTGCAACTCGACTCCATGAAGTCGGAATACG
\end{abstract}

$16 \mathrm{~S}$ rRNA gene sequences were compared with the available sequences in the databank with help of BLAST homology search and the isolate was found to be Salmonella enterica (Figure 1) and most similar to Salmonella enterica subsp. enterica serovar Panama str. ATCC 7378, complete genome (Sequence ID: $\mathrm{gb} \mid \mathrm{CP} 012346.1)$ and the next closest homologue was found to be Salmonella enterica subsp. enterica serovar Senftenberg genome assembly NCTC10384, chromosome: 1 (Sequence ID: emb|LN868943.1.). The sequences of strains were submitted to NCBI Gene Bank database under accession numbers KY604950.

\section{Phylogenetic analysis and elucidation of rRNA secondary structure}

In order to understand the significance in predicting the stability of chemical or biological molecules or entities of Salmonella enterica, RNA secondary structure prediction has been performed. The 16S RNA gene sequence obtained was used to deduce the secondary structure of RNA ViennaRNA Web Services (Figure 2). This server provides programs, web services, and databases, related to our work on RNA secondary structures. For thermodynamic structure prediction, RNAfold web server was used. 
The RNAfold web server will predict secondary structures of single stranded RNA or DNA sequences and current limits are $7,500 \mathrm{nt}$ for partition function calculations and
$10,000 \mathrm{nt}$ for minimum free energy only predictions. The free energy of secondary structure rRNA of Salmonella enterica was $477.40 \mathrm{kcal} / \mathrm{mol}$.

Fig.1 Phylogenetic tree of the isolate

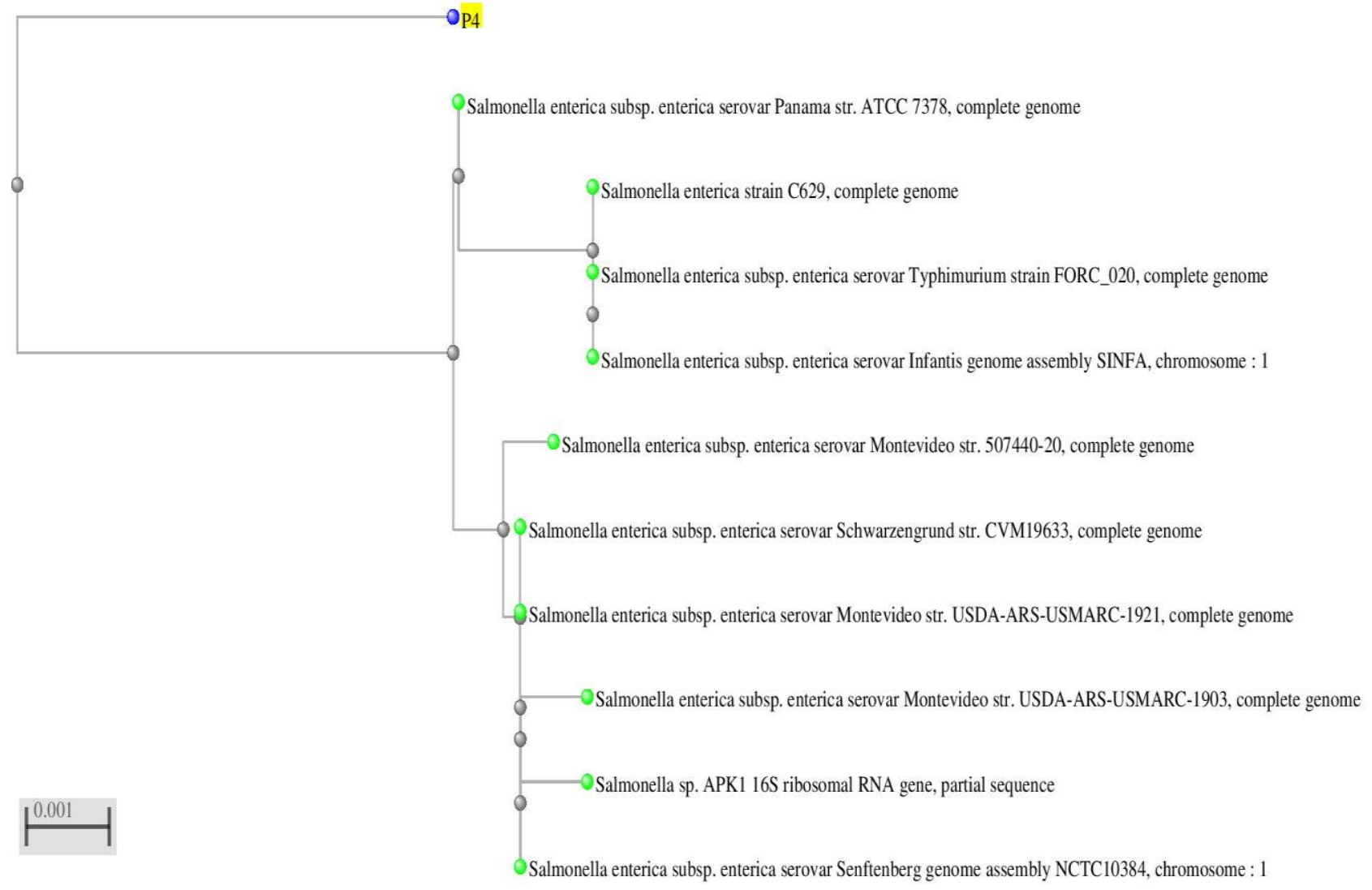


Fig.2 Secondary structure of rRNA of the isolate

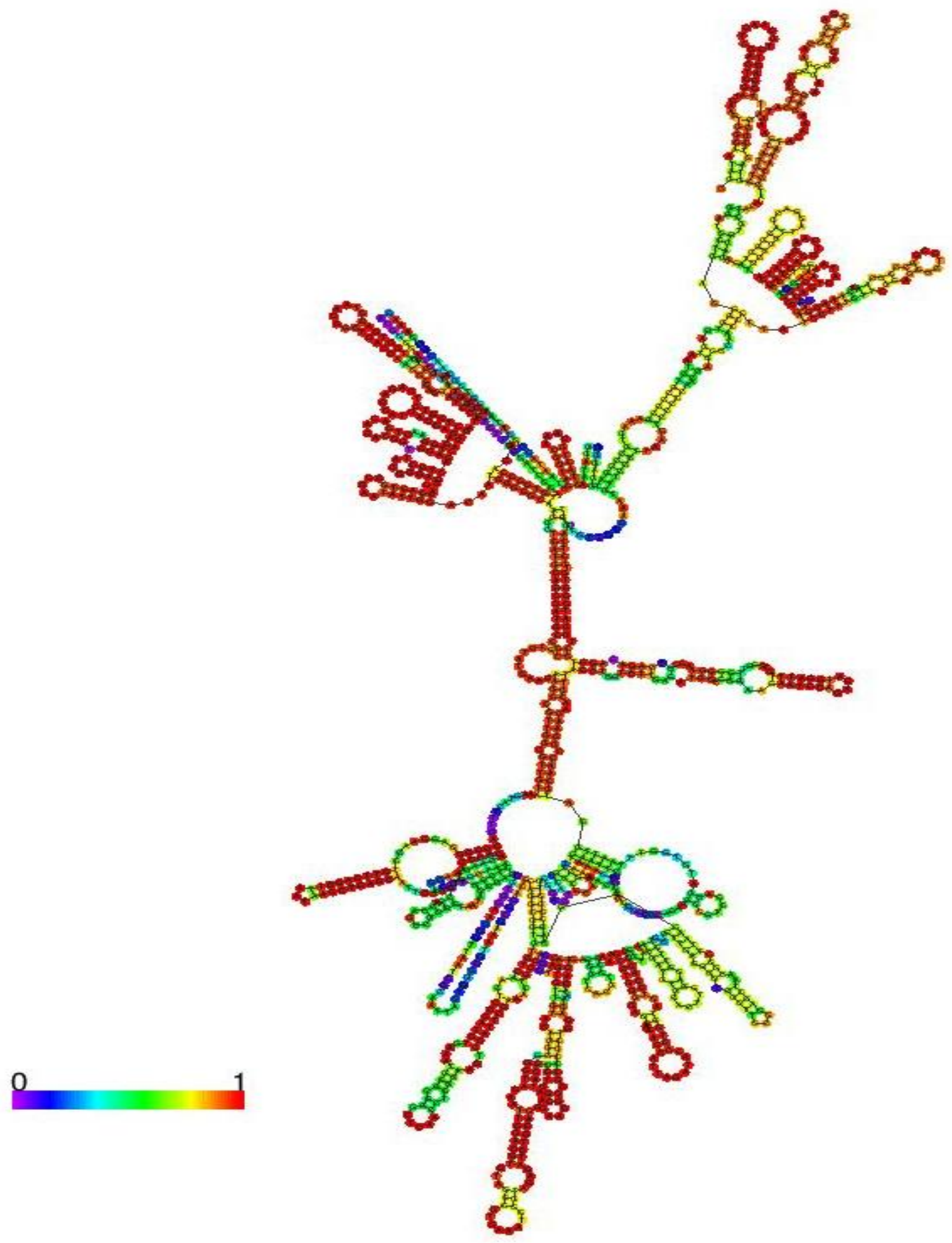


Table.1 Biochemical characteristics of the isolate

\begin{tabular}{lc}
\hline \multicolumn{1}{c}{ Biochemical tests } & Results \\
\hline Catalase & + \\
Nitrate reductase & + \\
Oxidase & - \\
Urease & - \\
Indole & - \\
Methyl Red & + \\
Voges-Proskauer & - \\
Citrate utilization & - \\
Glucose & + \\
Lactose & - \\
Sucrose & - \\
Gas production & - \\
H2S production & + \\
Gelatin Liquefaction & - \\
\hline
\end{tabular}

Fig.3 Mountain plot of secondary structure of rRNA of the isolate, is representation of the minimum free energy (MFE) structure, the thermodynamic ensemble of RNA structures, and the centroid structure and the positional entropy for each position

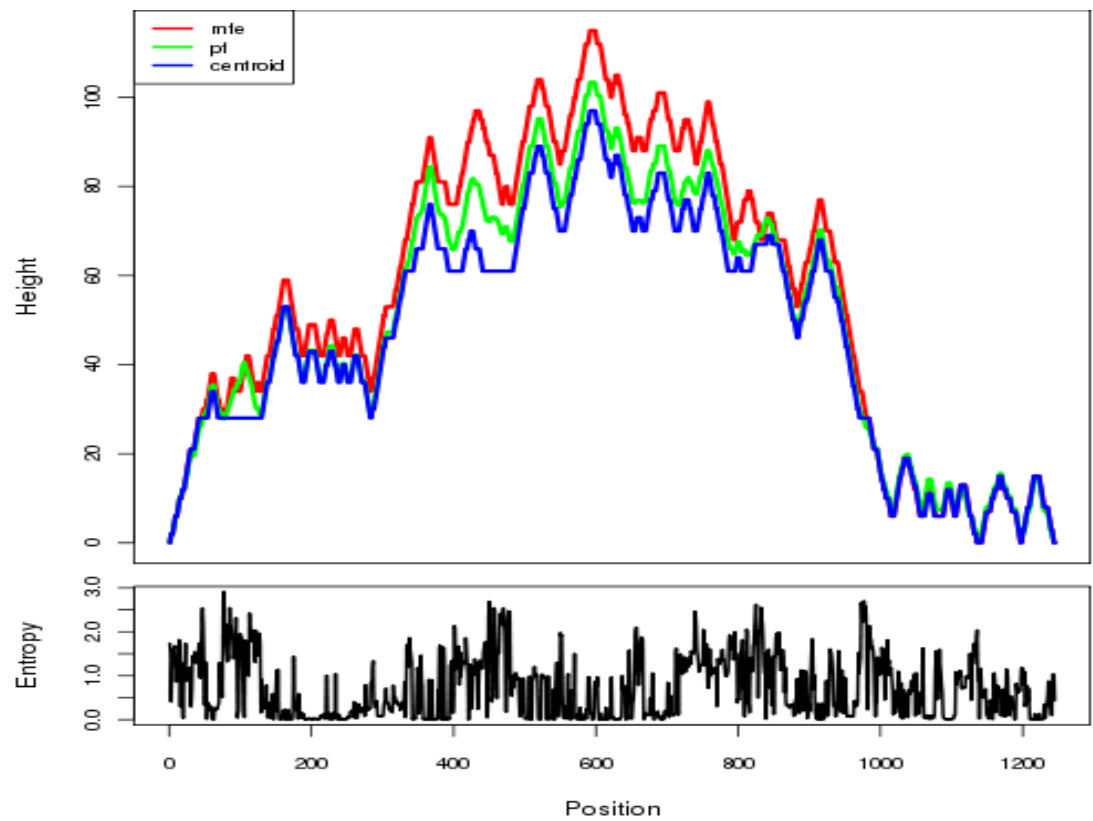

Mountain Plot was also drawn by the RNAfold web server which helps in predicting the hierarchical organization of RNA secondary structure, as nested helices translate into stacking mountains, easing the visual segmentation into domain. Here the sequence is drawn linearly, but this representation also presents, at each position $i$, the number of base-pairs nesting the position, i.e. involving bases respectively before and after $i$. In this setting, helices give rise to mountains while terminal loops translate into peaks (Figure 3). 
In conclusion, molecular techniques utilizing polymerase chain reaction (PCR), alone or in combination with DNA sequence analysis, have become increasingly popular in determining the evolutionary relationships of bacteria. With respect to Salmonella phylogenetics, these methods include sequence determination of the small subunit ribosomal RNA (rRNA or 16S rRNA, large subunit (23S rRNA) and various housekeeping (atpD, gapA, gyrB, mdh) or virulence (sefA, sopE), inv-spa region genes. Phylogenetic analyses have been used to understand the emergence and spread of pathogenic organisms, including Salmonella, within human populations and in epidemiological investigations. No universally accepted sequencing method exists for Salmonella. Currently, several promising new methods for identifying serotypes are emerging, based on single nucleotide polymorphisms. However, with respect to this study, the elucidation of rRNA secondary structure is documented for the first time and the $16 \mathrm{~S}$ rRNA sequence reported in the study may help other researchers for the identification and characterization of the Salmonella strains.

\section{References}

Ahmed, O.B., Asghar, A.H., Abd El-Rahim, I.H. and Hegazy, A.I. 2014. Detection of Salmonella in Food Samples by Culture and Polymerase Chain Reaction Methods. J. Bacteriol. Parasitol., 5: 187. doi: 10.4172/2155-9597.1000187

Cappuccino, J.G. and Sherman, N. 2004. Microbiology- A Laboratory Manual $6^{\text {th }}$ Edition. pp 132-185. Pearson Education
(Singapore) Pvt. Ltd., Indian Branch, New Delhi, India.

Chandra, P., and Chandra, A. 2016. Elucidation of rRNA secondary structure and phylogenetic analysis of plant growth promoting Streptomyces sp. based on $16 \mathrm{~s}$ RNA. $J$. Wheat Res., 8(2): 61-65.

Chen, J., Zhang, L., Paoli, G.C., Shi, C., Tu, S.I., Shi, X. 2010. A real-time PCR method for the detection of Salmonella enterica from food using a target sequence identified by comparative genomic analysis. Int. J. Food Microbiol., 137: 168-174.

10.1016/j.ijfoodmicro.2009.12.004

Godkar, P.B. 1999. Textbook of Medical Laboratory Technology. pp 338-361. Bhalani Publishing House, Mumbai, India.

Kaur, G. 2010. Evaluation of Some Traditional Medicinal Plants for their Antibacterial Activity. Ph D Thesis, Guru Nanak Dev University, Amritsar, Punjab, India.

Leekitcharoenphon, P., Hendriksen, R.S., Le Hello, S., Weill, F.X., Baggesen, D.L., Jun, S.R., Ussery, D.W., Lund, O., Crook, D.W., Wilson, D.J. and Aarestrup, F.M. 2016. Global genomic epidemiology of Salmonella enterica serovar Typhimurium DT104. Appl. Environ. Microbiol., 82: 2516-2526. doi:10.1128/AEM.03821-15.

Porwollik, S., J. Frye, L. D. Florea, F. Blackmer, and M. McClelland. 2003. A non-redundant microarray of genes for two related bacteria. Nucleic Acids Res., 31: 1869-1876.

Swaminathan, B., T.J. Barrett, S.B. Hunter, and R.V. Tauxe. 2001. PulseNet: the molecular subtyping network for foodborne bacterial disease surveillance, United States. Emerg. Infect. Dis., 7: 382-389.

Trkov, M., and Avgustin, G. 2003. An Improved 16S rRNA Based PCR Method for the Specific Detection of Salmonella enterica. Int. J. Food Microbiol., 80(1): 67-75.

\section{How to cite this article:}

Priyanka Chandra and Amaresh Chandra. 2017. Elucidation of rRNA Secondary Structure and Phylogenetic Analysis of Salmonella enterica based on 16S RNA. Int.J.Curr.Microbiol.App.Sci. 6(7): 4056-4063. doi: https://doi.org/10.20546/ijcmas.2017.607.420 